\title{
User Experience Evaluation in Entertainment and Games
}

\author{
Regina Bernhaupt \\ IRIT, Groupe ICS; 118 Route de Narbonne, \\ 31062 Toulouse, France \\ Regina.Bernhaupt@irit.fr
}

\begin{abstract}
In a nutshell. This tutorial comprehensively covers important user experience (UX) evaluation methods and opportunities and challenges of UX evaluation in the area of entertainment and games. The course is an ideal forum for attendees to gain insight into state-of-the art user experience evaluation methods, going way-beyond standard usability and user experience evaluation approaches in area of human-computer interaction. It surveys and assesses the efforts of user experience evaluation of the gaming and human computer interaction communities during the last 10 years.
\end{abstract}

Keywords: entertainment, user experience, evaluation methods, beyond usability, game.

\section{Introduction}

User experience evaluation in games and more general in interactive entertainment system has become a focus of (research) attention in the areas of human-computer interaction (HCI). During the last 10 years the scientific communities of HCI and game research were starting to learn from each other: On the one hand UX evaluation methods from HCI are used during the game development, on the other side HCI was borrowing and investigating aspects of the gaming experience like immersion, flow or fun, to better understand the concept of UX [1].

This course is about evaluating user experience in the area of entertainment and (video) games. It provides an overview on what user experience is about (contrary to usability), it provides an understanding on what makes for successful future games and entertainment experiences and what set of user experience evaluation methods is currently available and used for the development of games.

Objective of this course is to provide an overview on user experience evaluation in the games and entertainment area. The goal is to provide some definitions of user experience, explain the factors that might be contribution to the overall user experience in a game (e.g. flow, immersion, playability) and (using a set of examples) to explain how games development is slightly different from standard software engineering development. Based on these foundations the objective is to give an overview on existing methods from the field and allow participants in the course a first hands-on experience trying out how to apply one of the methods to a real game. 


\section{Schedule and Presentation Format}

The tutorial will use both presenter-led material and group-based exercises throughout the sessions. Small exercises and hands-on examples will encourage attendees both to critique existing approaches as well as posing their own view on the relative importance of the various factors that are contributing to user experience. The course will include demonstrations of UX evaluation methods and use video clips to show the variety of applications domains the methods can be applied to. Course materials will include copies of the presentation materials as well as extensive follow-up reading lists.

The tutorial will run over a half day. The material will focus on an overview on available evaluation methods, their application in the game-development life cycle and shortcomings and benefits for each of the methods. The course consists of four blocks:

1. Introduction to the topic, definition of user experience in entertainment and games, application areas

2. Overview on user experience evaluation methods

3. Application of user experience evaluation (hands-on exercise) and wrap up of insights from the exercise

4. Key insights summary and lessons learned

\section{Target Audience}

This course will appeal to a broad audience. For developers and designers: the course will help understand how to evaluate user experience in the area of games and entertainment and how outcomes of the evaluation can be integrated in the next iteration of the game and entertainment application development; for industrial and academic researchers: The course will provide an overview on current methods in that area, and can help understand the concept of user experience; for students: the course provides a first introduction to user experience in games, but lessons can be taken up for the application in other domains (e.g. definition of UX, application of methods during a development life cycle). The course additionally can be of interest to any attendee of Interact who wants to get a first idea and understanding on user experience and user experience evaluation in general.

\section{References}

1. Bernhaupt, R.: User Experience Evaluation in Games: Concepts and Methods. Springer, Heidelberg (2010) 